\title{
Simulation Package based on PLACET
}

\author{
E. T. d'Amico, G. Guignard, N. Leros, D. Schulte, CERN, Geneva, Switzerland
}

\begin{abstract}
The program PLACET is used to simulate transverse and longitudinal beam effects in the main linac, the drive-beam accelerator and the drive-beam decelerators of CLIC, as well as in the linac of CTF3. It provides different models of accelerating and decelerating structures, linear optics and thin multipoles. Several methods of beam-based alignment, including emittance tuning bumps and feedbacks, and different failure modes can be simulated. An interface to the beam-beam simulation code GUINEA-PIG exists. Currently, interfaces to MAD and TRANSPORT are under development and an extension to transfer lines and bunch compressors is also being made. In the future, the simulations will need to be performed by many users, which requires a simplified user interface. The paper describes the status of PLACET and plans for the future.
\end{abstract}

\section{INTRODUCTION}

The Compact Linear Collider CLIC is based on a twobeam scheme in which the power necessary for the mainbeam acceleration is provided by the deceleration of the drive beam [1],[2]. On the one hand, the main-beam parameters have to satisfy very stringent conditions so that the beam quality is preserved during transfer and acceleration. On the other hand, decelerating the drive beam implies important challenges associated with a strong energy spread and a very large number of high-charge bunches. Only a simulation program may convince the designing team that these can be achieved, at least in principle. Moreover the new CLIC Test Facility (CTF3) [3] is a complex accelerator on its own, which is intended to prove the validity of the proposed RF Power Source [4] and needs extensive simulation of its fully loaded linac. The simulations include the wake-field effects, the trajectory corrections and specific characteristics of the decelerated beam, such as chromaticity in a bunch and in a train. Feedbacks are envisaged to compensate ground motion effects. Each feedback consists of two focusing quadrupoles and three beam position monitors (BPM) which detect and correct the beam position and angle deviation from the trajectory obtained by the last linac alignment. To these ends, a new program was developed which is called PLACET (Program for Linear Accelerator Correction Efficiency Tests) [5] and for which a brief description can be found in Section 2. This tracking code has since also been used to simulate some particular effects in TESLA [6]. It is written in an efficient computer language, and its high modularity combined with a strong hierarchical structure allows additions of interfaces to other programs in a relatively easy way, as for example the link to the beam-beam simulation code GUINEA-PIG [7]. These interfaces are described in Section 3. An example of how a very complex system like the feedbacks can be inserted in PLACET while taking advantage of its power will be found in Section 4. The flexibility of such package opens the way to further improvements in user-friendliness and in adapting to new beam-line configurations, some of which are treated in Section 5.

\section{SHORT DESCRIPTION OF PLACET}

The program PLACET is written in $\mathrm{C}$ with a few commands of $\mathrm{C}++$. It may be considered as an extension of the widely used Tcl shell, some graphics facilities being provided by the Tk toolkit. About 120 new Tcl commands link easily and dynamically more than 800 routines written in C. Commands span all levels of complexity, from just moving a single component to the simulation of a full trajectory correction or of several feedbacks in the linac. An example of the display created by the command "BeamlineView" is shown in Fig. 1. The position of an element can be obtained by clicking on it, and the whole linac can be scrolled horizontally.

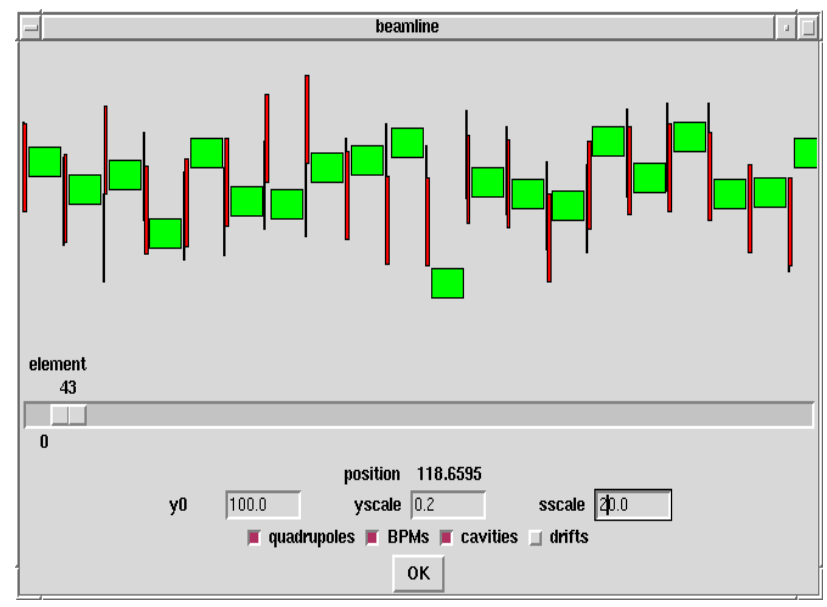

Figure 1: Display created by the command "BeamlineView".

With this program it is possible to simulate the dynamics of a beam in the accelerating or decelerating CLIC linacs in the presence of strong transverse and longitudinal wakefields, including beam loading effects [8] [9]. Beam-based alignment procedures can be carried out assuming phase errors, misalignments of beam-line elements, ground motion, earth's magnetic field and beam jitter. The main elements like quadrupoles (considered as thick lenses) and several types of RF structures (either accelerating ones with 
relatively low group velocities or decelerating ones with high group velocity) are fully simulated. The sophisticated schemes which have been developed to correct the beam trajectory have also been included, in order to perform extensive tests of the emittance growth averaged over a large number of simulated CLIC machines. Among others, the ballistic correction [10] is implemented as well as a version based on trajectory difference measurement, called Multistep Lining-up [11]. Another useful feature is to save corrected linacs, and after having reloaded one of them, it is possible to modify at will the RF phase and amplitude of each structure, the strength of each quadrupole, the position of each element and the bunch properties. The simulation of a single bunch beam, sliced longitudinally into 21 slices and running through a $1.5 \mathrm{TeV}$ linac with $22000 \mathrm{RF}$ structures and 1324 quadrupoles, is done on a $600 \mathrm{MHz}$ Pentium III. The average computing times over 100 simulations with randomly modified misalignments are $267 \mathrm{~s}$ and $162 \mathrm{~s}$ for a one-to-one correction and a ground motion correction done by 40 feedbacks respectively.

\section{INTERFACES}

In colliders with a high disruption parameter, beambeam effects are strongly modified by the actual distribution of the particles within the bunch and their offsets at the end of the linac. The consequence on the performance or emittance growth has therefore to be evaluated in a way which effectively reflects this distortion. The chosen procedure consists of two steps. In the first one, the beam is tracked by the code PLACET through the full length of the linacs, a virtual matching insertion being added to each of them in order to get the nominal optics at the interaction point (IP). In the second step, the code GUINEA-PIG uses these simulated beams at IP to estimate the beam-beam effect. The first results show that the consequences are significant for TESLA [6] and that they should not be neglected in CLIC.

The method was made applicable by developing an interface inside PLACET. The results obtained are described at length in a paper presented in this conference [6]. Similarly a new interface has been added which links PLACET to the simulation code SIXTRACK [12] via an intermediate file that can be easily extended also as input to MAD [13] and to TRANSPORT [14] codes. These facilities have shown that it was possible to incorporate new routines inside PLACET in a relatively short time and without requiring the complete knowledge of the program details.

\section{APPLICATION TO FEEDBACKS}

The future linear collider has very tight tolerances on the static and dynamic alignment of the beam-line. PLACET offers the possibility to simulate various misalignment sources and to use different correction techniques like feedbacks which steer the beam back to its original trajectory.

Two feedback models are available at present. We can specify a list that gives the position, the number of BPM's and the gain of each feedback.

The first model called "perfect feedbacks", re-steer the beam in a single pulse to its trajectory by compensating exactly the position and angle errors due to ground motion movements occurring upstream of each feedback. Errors in BPM readings are neglected. In the code, the same pulse is used for correction and tracking. Displacements occurring during the corrections are neglected and thus the set of "perfect feedbacks is an optimistic model".

The second model called "independent feedbacks", which is more realistic, has been implemented recently. Since each feedback does not take advantage of the information provided by the other feedbacks, the set of independent feedbacks represents a pessimistic model. An RMS error of $100 \mathrm{~nm}$ for the BPM readings is now taken into account. Thus only a fraction, called gain $\mathrm{g}$, of the computed correction is actually applied. The optimum gain depends on the misalignment model. Considering 40 independent feedbacks, it has been found that the optimum gain to correct the ground motion effect is 0.02 [15]. It may be observed in Fig. 2 that the optimum gain is 0.075 if the quadrupoles move according to a random walk occurring from pulse to pulse, the amplitude of the displacement being Gaussian with $\sigma=1 \mathrm{~nm}$.

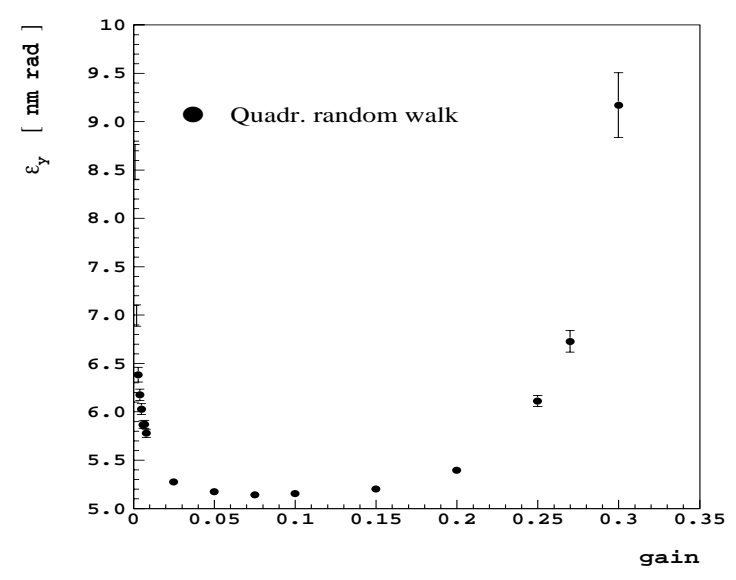

Figure 2: Vertical emittance growth (CLIC, $E_{C M}=3 \mathrm{TeV}$ ) versus the gain due to a pulse to pulse random walk of quadrupoles ( $\sigma=1 \mathrm{~nm}$ ) corrected by 40 independent feedbacks.

In order to get a realistic emittance growth during the correction, different misalignments may be simulated from pulse to pulse, such as beam jitter, quadrupole jitter, ground motion (described by the ATL model with $\mathrm{A}=0.5 \times 10^{-6}$ $\left.(\mu \mathrm{m})^{2} /(\mathrm{sm})\right)$, random walk and specific displacements of some predefined elements. Changes of RF-phase, RFamplitudes and bunch characteristics can also be taken into account. A very useful feature adds the pulse to pulse time evolution of the emittance due to dynamic changes. The number of pulses to simulate (or the time interval for the correction) has to be set, and the time evolution of the emit- 
tance is easily performed as an average over many different machines.

A full discussion related to perfect and independent feedbacks can be found in [15]. The emittance growth due to a random walk of quadrupoles (as described above) is shown in the Fig. 3, where no correction is applied in one curve and the other curves correspond to different gains for a correction done by a set of 40 independent feedbacks. The exchange of informations about the nominal lattice between feedbacks has also been implemented to improve this model.

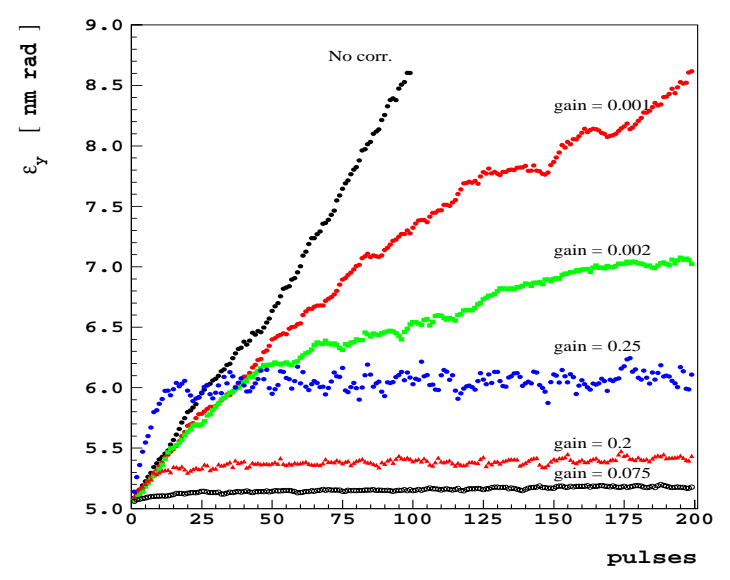

Figure 3: Time evolution of the vertical emittance (CLIC, $3 \mathrm{TeV}$ ) due to a pulse to pulse random walk of quadrupoles $(\sigma=1 \mathrm{~nm})$.

\section{FUTURE PLANS AND IMPROVEMENTS}

The program architecture of PLACET opens a wide range of applications. Its strongest feature is the ease with which hooks to other simulations programs can be built and the simple implementation of new beam-line elements. The latter will be further enhanced by introducing some features of object-oriented programming like inheritance and encapsulation. The final goal is to provide a tool that may help the joint efforts from various areas of the CLIC study to simulate the behaviour of the beam in diverse sub-systems. This requires adding facilities and interfaces to other codes widely used either for injection systems or for beam delivery studies. The first ones on the priority list are the bunch compressors to give the capability of studying the beam behaviour in the longitudinal plane and to assess the effects of coherent radiation by interfacing to specialised codes like $\operatorname{TraFiC}^{4}$ [16]. Future developments will include the fact that there are several areas of the CLIC complex, beside the main- and drive-linacs mentioned above, where simulations are required: they concern the whole drive-beam generation system (acceleration linacs, bunch compressors, transfer lines and bends, com- pressor rings), and important parts of the main-beam preacceleration system (low-frequency linacs, bunch compressors, transfer lines, large loops). In addition, beam tracking is also essential for CTF3. At the same time the userfriendliness will be improved by taking advantage of the full power of the script language Tcl/Tk. The user guide which is still in a draft stage is available on the Web and will be updated regularly to keep track of the program evolution. Finally it is intended to comply with the Extended Standard Input Format of accelerator beam-lines [17] to further open PLACET to other users and applications.

\section{REFERENCES}

[1] J.-P. Delahaye and 26 co-authors, The CLIC Study of a Multi-TeV $e^{+} e^{-}$Linear Collider, Proc. PAC 99, New York (1999).

[2] CLIC Study Team, A $3 \mathrm{TeV}$ Linear Collider Based on the CLIC Technology, G. Guignard editor, CERN 2000-008 (2000).

[3] CLIC Study Team, Proposal for Future CLIC Studies and a New CLIC Test Facility (CTF3), CLIC Note 402, Geneva (1999).

[4] H. H. Braun and 15 co-authors, The CLIC RF Power Source, CERN report 99-06, Geneva (1999).

[5] D. Schulte, The Tracking Code PLACET, draft at http://home.cern.ch/dschulte/placet.html.

[6] R. Brinkmann, O. Napoly, D. Schulte, Beam-Beam instability Driven by Wakefield Effects in Linear Colliders, this Conference.

[7] D.Schulte, Beam-Beam Simulations with GUINEA-PIG, ICAP98, Monterey, Ca, USA, (1998).

[8] D. Schulte, Emittance Preservation in the Main Linac of CLIC, EPAC98, Stockholm (1998).

[9] A. Millich, A. Riche, D. Schulte, Beam stability in the Drive-Beam Decelerator of CLIC using Structures of HighOrder Symmetry, PAC99, New York, USA (1999).

[10] T. Raubenheimer, D. Schulte, The Ballistic Alignment Method, PAC99, New York, USA (1999).

[11] E. T. d'Amico, G. Guignard, Multi-Step Lining-up Correction of the CLIC trajectory, PAC99, New York, USA (1999).

[12] E. Forest and F. Schmidt, Map creation and analysis via overloaded tools in FORTRAN 90, EPAC 2000, Vienna (2000).

[13] H. Grote, The MAD Program User's Reference Manual, CERN/SL/90-13 (AP) Rev. 5, (1996).

[14] K. L. Brown et al., TRANSPORT: A Computer Program for Designing Charged Particle Beam Transport Systems, SLAC-Report-91 Rev. 2 (1977).

[15] N. Leros, D. Schulte, Dynamic Effects in the Main Linac of CLIC, this Conference.

[16] A.Kabel, M. Dohlus, T. Limberg, Using $\mathrm{TraFiC}^{4}$ to calculate and minimise emittance growth due to coherent synchrotron radiation, NIM in Physics Research Section A, Volume 455, Issue 1, (2000)

[17] D. C. Carey and F. C. Iselin, A Standard Input Language for Particle Beam and Accelerator Computer Programs, Proc. of the 1984 Summer Study, Snowmass, Colorado (1984). 